Courrier du Centre International BlaisePascal

38-39 | 2017-2018

Varia

\title{
Les errata des Nouveaux éléments de géométrie d'Antoine Arnauld
}

Dominique Descotes

\section{OpenEdition}

1 Journals

Édition électronique

URL : https://journals.openedition.org/ccibp/1493

DOI : 10.4000/ccibp.1493

ISSN : 2493-7460

Éditeur

Centre international Blaise Pascal

Édition imprimée

Date de publication : 1 janvier 2019

Pagination : 175-190

ISBN : 978-2-84516-897-8

ISSN : 0249-6674

Référence électronique

Dominique Descotes, « Les errata des Nouveaux éléments de géométrie d'Antoine Arnauld ", Courrier du Centre International Blaise-Pascal [En ligne], 38-39 | 2017-2018, mis en ligne le 21 juin 2021, consulté le 05 mai 2022. URL : http://journals.openedition.org/ccibp/1493; DOI : https://doi.org/10.4000/ccibp. 1493

Ce document a été généré automatiquement le 5 mai 2022.

Centre international Blaise Pascal 


\title{
Les errata des Nouveaux éléments de géométrie d'Antoine Arnauld
}

\author{
Dominique Descotes
}

1 On connaît la discussion qui opposa Blaise Pascal et Antoine Arnauld sur les mathématiques, et le défi amical qu'ils se sont lancé de composer des éléments de géométrie modernes propres à remplacer Euclide par des modernes. Jean Mesnard situe cet épisode vers 1655, contemporain de la rédaction de L'esprit géométrique et de l'Introduction à la géométrie.

2 De l'Introduction à la géométrie de Pascal ne subsiste qu'une page, copiée et commentée par Leibniz, qui dessine les voies de la géométrie de position. Elle n'a pour le moment fait l'objet que de deux études considérables ${ }^{1}$.

3 Arnauld a probablement composé un premier état de ses futurs Nouveaux éléments de Géométrie au Chesnay, au mois de janvier. Lorsque les deux amis confrontèrent leurs essais respectifs, ils attribuèrent la palme à l'ouvrage d'Arnauld, sans qu'il soit aujourd'hui possible de connaître les raisons de ce jugement passablement surprenant. Un état ancien de la Logique de Port-Royal contenu dans les recueils manuscrits Vallant, datant sans doute de 1659 ou 1661, montre que bientôt, Arnauld projeta de publier ses Éléments ${ }^{2}$. La première édition ne sort cependant chez Savreux qu'en 1667.

4 Mais comme l'écrit Pierre Nicole dans le second Discours introductif de la Logique, « les premières éditions des livres » doivent être considérées comme "des essais informes que ceux qui en sont les auteurs proposent aux personnes de lettres pour en apprendre leurs sentiments", en vue d'éventuelles corrections ${ }^{3}$. Seize ans plus tard, Arnauld publie une deuxième édition de son livre chez Desprez. Mais les conditions de travail qui s'offrent à lui en 1683 sont bien moins favorables qu'en 1667, à l'époque où la Paix de l'Église s'annonçait. Il se vit alors obligé de se cacher en exil. Cette nouvelle édition est profondément modifiée pour ce qui touche l'axiomatique : arguant du fait qu'il n'a jamais été satisfait de la théorie des raisons de sa première édition, Arnauld en a entièrement remanié les Livres II et III, qui développent une théorie fondamentale entièrement nouvelle, mais qui a laissé à peu près intact le reste de l'édifice ${ }^{4}$. 
Lorsque j'ai composé l'édition des Nouveaux éléments de géométrie, qui tient compte des deux versions publiées sous la responsabilité d'Arnauld ${ }^{5}$, j'ai rencontré à plusieurs reprises dans l'impression de 1667 des défauts qui tenaient, non à la substance mathématique de l'ouvrage, mais à son impression, notamment plusieurs figures manquantes ou erronées et des équations incompréhensibles. En fait, presque tous pouvaient être résolus à l'aide de l'édition de 1683: quoiqu'elle-même non indemne d'erreurs, surtout dans les références, elle avait l'avantage de fournir les figures manquantes et de corriger les fautes de 1667, de sorte que l'apparat critique suffisait pour établir un texte satisfaisant.

6 En 2017 la Bibliothèque du Patrimoine de Clermont-Ferrand a fait l'acquisition d'un exemplaire des Nouveaux éléments de 1667 qui a ajouté un épisode instructif et pittoresque à l'histoire de cet ouvrage, qui permet d'entrer dans les secrets de l'impression de l'ouvrage'.

7 Il suffisait du reste de consulter les tables des Fautes à corriger et des Fautes dans les figures placées à la suite du Privilège du roi dans l'édition de 1667, pour deviner que l'imprimeur avait manifestement reçu un manuscrit d'une lecture difficile: le prote avait lu moyen au lieu de maxime, même point au lieu de et du même côté, divisée en 6 et en $c$ au lieu de en $b$ et en $c$. Quant aux figures, ces errata signalaient que plusieurs manquaient, et que d'autres comportaient des imperfections ou des lettres mal placées. Les correcteurs indiquent du reste comment rectifier certaines erreurs à la plume.

Fig. 1. Fautes à corriger.

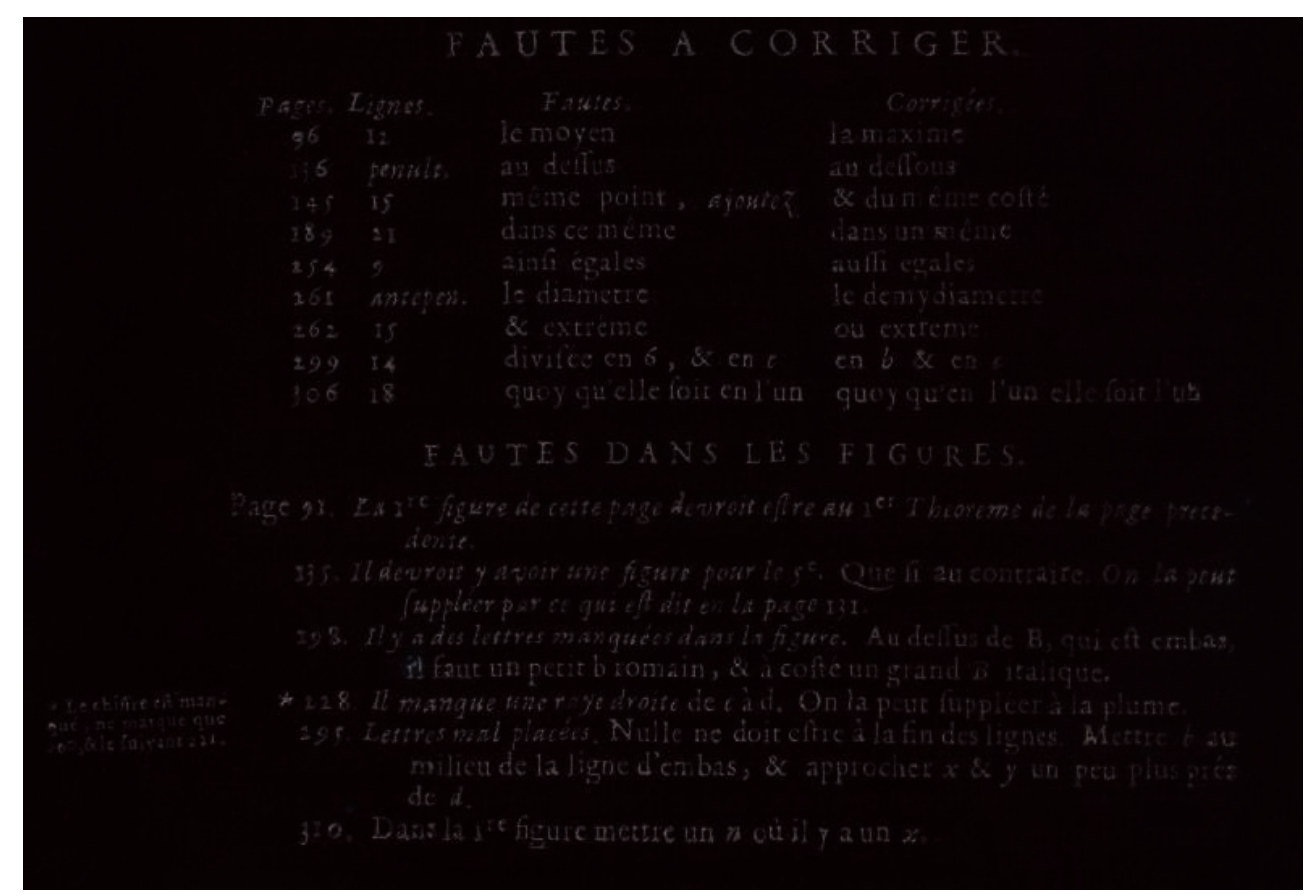

Bibliothèque du Patrimoine de Clermont-Métropole.

8 Il semble même que certaines corrections aient été ajoutées après coup, puisque l'une d'elles est placée en marge de la page, avec une étoile pour indiquer sa place.

9 Rien en tout cela qui ne soit au fond assez ordinaire. Mais l'acquisition de la Bibliothèque de Clermont permet d'entrer de manière beaucoup plus profonde dans les 
tribulations secrètes de l'édition de 1667. Visiblement, les « fautes à corriger » n'ont pas suffi pour rendre l'ouvrage irréprochable.

\section{Modification d'une démonstration}

10 L'exemplaire de Clermont révèle un fait que les exemplaires ordinaires des Nouveaux éléments de 1667 occultent: la suppression de quatre pages qui se trouvaient dans l'impression initiale, compensée par la substitution par quatre autres. Ces quatre pages, qui appartiennent à la même feuille du même cahier, portent les numéros 199 (recto) et 200 (verso), et 251 (recto) et 252 (verso). Dans la plupart des exemplaires, une fois la feuille ancienne supprimée, détruite et remplacée, la substitution devient naturellement invisible. Mais dans l'exemplaire de Clermont, pour une raison inconnue, les pages anciennes sont demeurées en place ; et corrélativement, celles qui contiennent la rédaction définitive ont été reliées en un autre endroit, vers la fin du volume. De sorte que, par une chance extraordinaire, le volume contient les deux impressions.

11 Les feuillets supprimés sont appelés cancellanda (à supprimer) s'ils sont restés pris dans l'ouvrage; ils sont dits cancellata (supprimés) s'ils ont disparu. Les feuillets qui les remplacent sont dits cancellantia (supprimants).

12 Sur l'exemplaire qui nous intéresse, les cancellanda, qui devraient avoir été supprimés, se trouvent encore à leur place originelle du livre. En revanche, les cancellantia, qui sont appelés à remplacer les précédents et à devenir définitifs, se trouvent reliés entre les pages 338 et 339, dans la partie relative aux carrés magiques ${ }^{7}$. L'état de la composition du livre que nous envisageons est donc antérieur à la mise en ordre définitive de la pagination.

13 Les pages 199 et 200 ne nous intéressent pas : elles n'ont fait l'objet d'aucune retouche, et n'ont été changées que parce qu'elles étaient matériellement associées aux pages 251 et 252 sur une seule et même feuille.

14 En revanche, les deux autres pages ont subi une importante refonte, pour les figures et pour le texte. Elles appartiennent au Livre XII, Des figures en général considérées selon leurs angles et leurs côtés, dans un ouvrage qui en comprend quinze.

15 La p. 251 initiale (désignons-la par le signe 251-1), contenait la section consacrée aux "figures inscrites ou circonscrites au cercle". Après les deux paragraphes "Des inscrites ", se présente un Second théorème, $\mathrm{n}^{\circ} \mathrm{XV}$. On note que cette page ne contient qu'une seule figure, un hexagone régulier. 
Fig. 2. Page 251-1

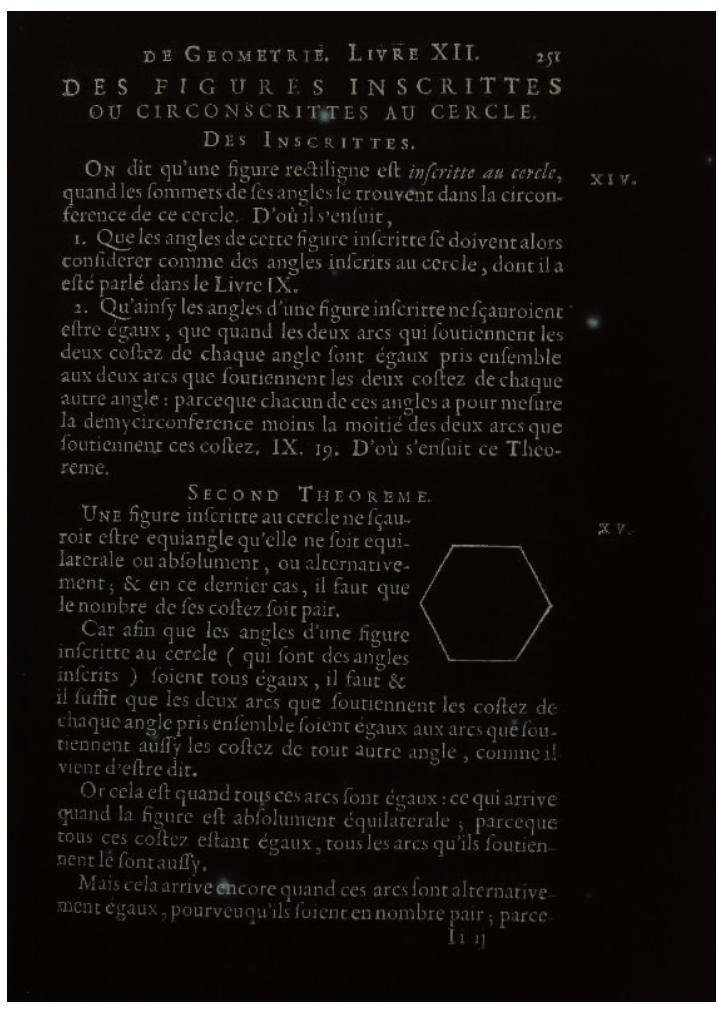

Bibliothèque du Patrimoine de Clermont-Métropole.

\section{Le texte va comme suit :}

Une figure inscrite au cercle ne saurait être équiangle qu'elle ne soit équilatérale ou absolument, ou alternativement; et en ce dernier cas, il faut que le nombre de ses côtés soit pair.

Car afin que les angles d'une figure inscrite au cercle (qui sont des angles inscrits) soient tous égaux, il faut et il suffit que les deux arcs que soutiennent les côtés de chaque angle pris ensemble soient égaux aux arcs que soutiennent aussi les côtés de tout autre angle, comme il vient d'être dit

Or cela est quand tous ces arcs sont égaux: ce qui arrive quand la figure est absolument équilatérale; parce que tous ces côtés étant égaux, tous les arcs qu'ils soutiennent le sont aussi.

Mais cela arrive encore quand ces arcs sont alternativement égaux, pourvu qu'ils soient en nombre pair ; parce

17 Le texte continue sur la page 252 (que nous désignons par le sigle 252-1). 
Fig. 3. Page 252-1

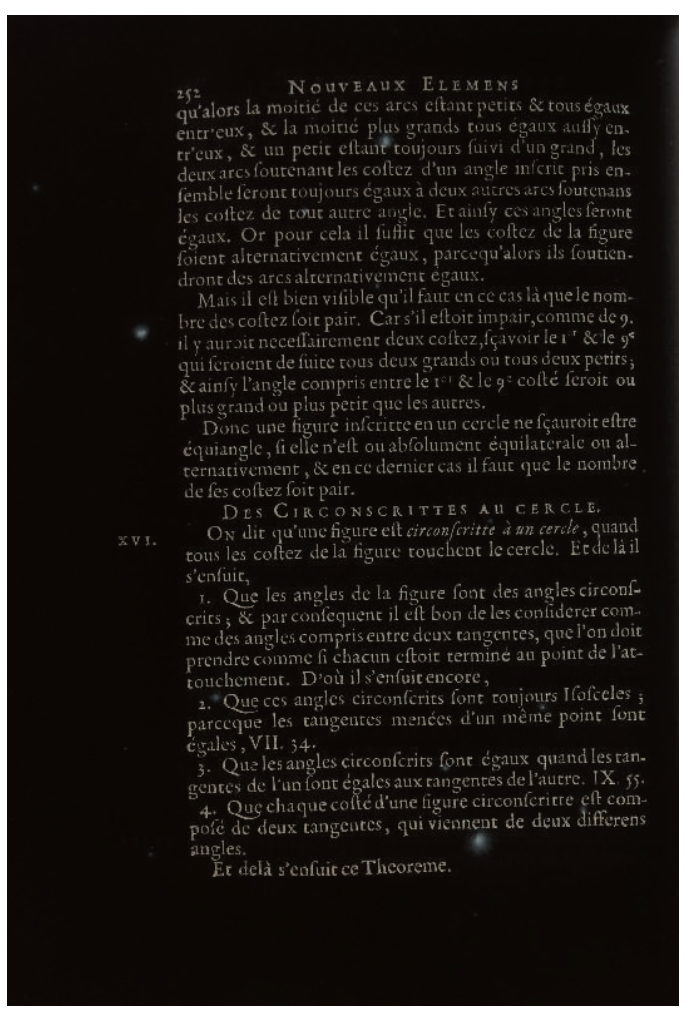

Bibliothèque du Patrimoine de Clermont-Métropole.

Dans la transcription ci-dessous, nous mettons en italique la partie qui nous intéressera par la suite :

qu'alors la moitié de ces arcs étant petits et tous égaux entr'eux, et la moitié plus grands tous égaux aussi entr'eux et un petit étant toujours suivi d'un grand, les deux arcs soutenant les côtés d'un angle inscrit pris ensemble seront toujours égaux à deux autres arcs soutenant les côtés de tout autre angle. Or pour cela il suffit que les côtés de la figure soient alternativement égaux, parce qu'alors ils soutiendront des arcs alternativement égaux.

Mais il est bien visible qu'il faut en ce cas là que le nombre des côtés soit pair. Car s'il était impair, comme de 9, il y aurait nécessairement deux côtés, savoir le $1^{\text {er }}$ et le $9^{e}$ qui seraient de suite tous deux grands ou tous deux petits; et ainsi l'angle compris entre le $1^{\text {er }}$ et le ge côté serait ou plus grand ou plus petit que les autres.

Donc une figure inscrite en un cercle ne saurait être équiangle, si elle n'est ou absolument équilatérale ou alternativement, et en ce dernier cas il faut que le nombre de ses côtés soit pair.

19 La raison pour laquelle le texte a été modifié est visible. La figure de la p.251-1 ne correspond pas au texte qu'elle est censée illustrer : elle représente bien un polygone, mais non le cercle circonscrit auquel se rapporte le texte. D'autre part, ce théorème exige deux figures différentes, l'une avec un polygone régulier, l'autre doté des côtés alternativement égaux, et non pas une seule.

20 Arnauld est sans doute intervenu auprès de l'imprimeur Savreux, pour faire remplacer la figure incorrecte par deux autres correctes, savoir des polygones inscrits au cercle, l'un équilatéral, l'autre doté de côtés égaux alternativement ${ }^{8}$.

21 Mais deux figures occupent plus de place qu'une seule. 
22 Savreux semble avoir concédé la correction, probablement parce qu'il a dû admettre qu'il était responsable de la faute (nous verrons plus bas que ce n'est pas toujours le cas). Mais il n'a pas voulu réimprimer les pages suivantes, qui avaient sans doute été déjà composées. Il a donc proposé (ou imposé) d'abréger le texte pour laisser place à deux figures nouvelles.

23 Voici ces deux pages définitives, telles qu'elles se présentent, après correction, dans les éditions ordinaires.

Fig. 4. Page 251-2

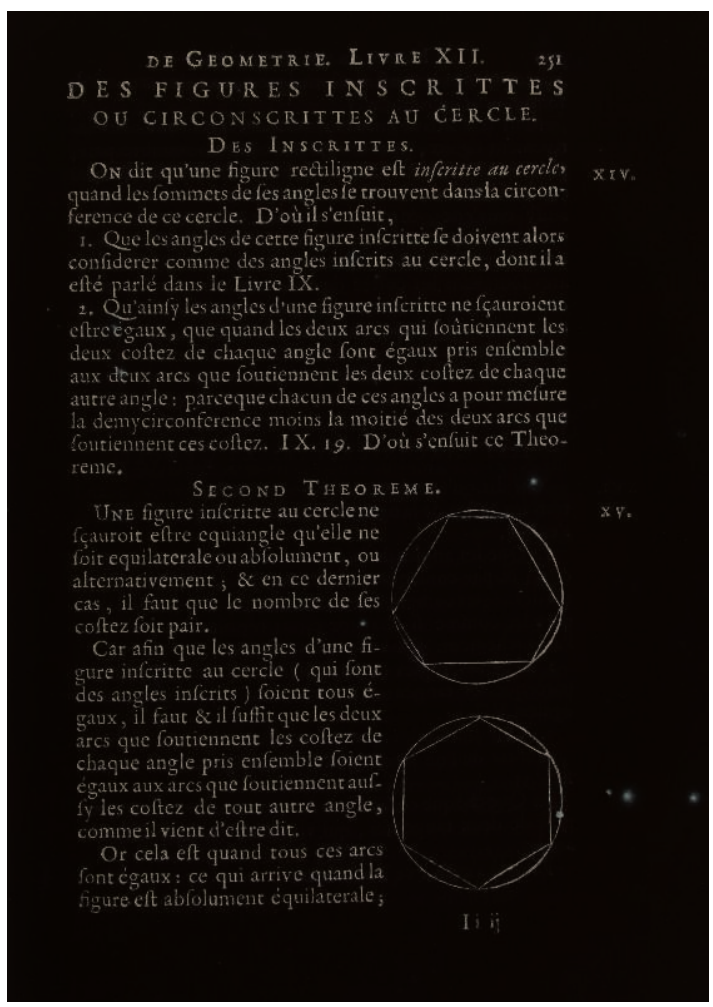

Bibliothèque du Patrimoine de Clermont-Métropole. 
Fig. 5. Page 252-2

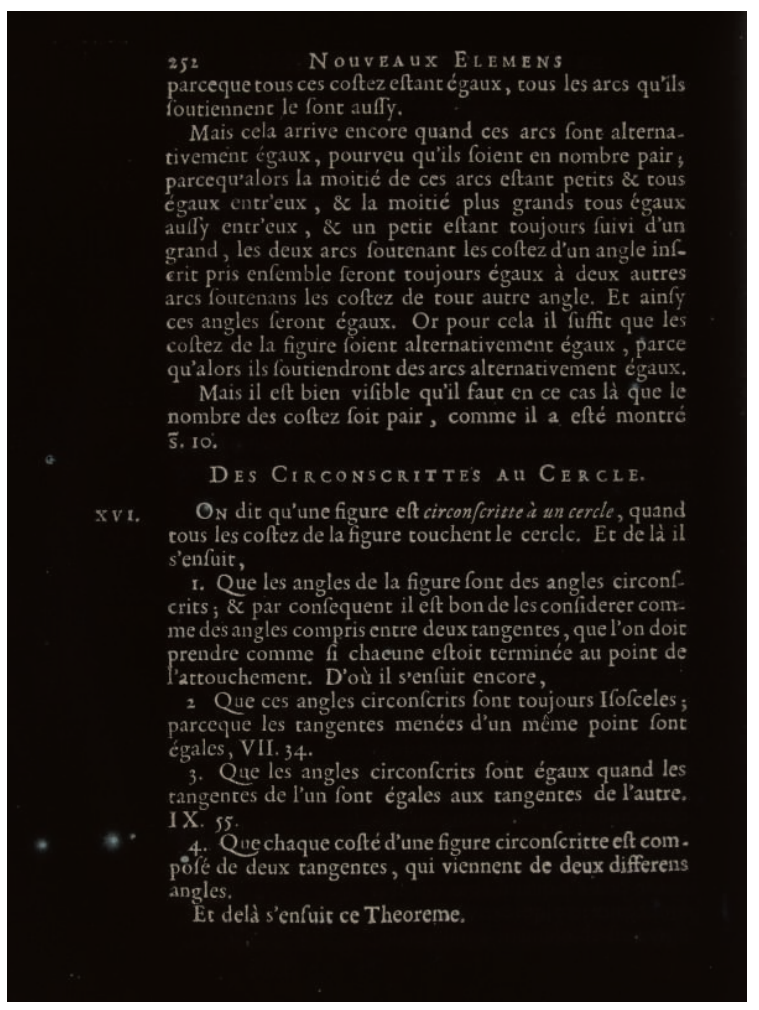

Bibliothèque du Patrimoine de Clermont-Métropole.

Toute la partie de 252-1 qui montre la nécessité d'avoir un nombre de côtés pair dans le cas d'un polygone dont les côtés sont alternativement égaux a été supprimée. Reste le texte suivant sur la p. 252-2:

parce que tous ces côtés étant égaux, tous les arcs qu'ils soutiennent le sont aussi. Mais cela arrive encore quand ces arcs sont alternativement égaux, pourvu qu'ils soient en nombre pair; parce qu'alors la moitié de ces arcs étant petits et tous égaux entr'eux, et la moitié plus grands tous égaux aussi entre eux, et un petit étant toujours suivi d'un grand, les deux arcs soutenant les côtés d'un angle inscrit pris ensemble seront toujours égaux à deux autres arcs soutenant les côtés de tout autre angle. Et ainsi ces angles seront égaux. Or pour cela il suffit que les côtés de la figure soient alternativement égaux.

Mais il est bien visible qu'il faut en ce cas là que le nombre des côtés soit pair, comme il a été montré $\hat{s} 10$.

La partie écartée établit la nécessité que, dans le cas d'un polygone dont les côtés sont alternativement égaux, leur nombre soit pair.

L'imprimeur ne saurait avoir effectué cette modification de son propre chef. Seul Arnauld a pu fournir le moyen de compenser la suppression par une référence convenable, « comme il a été montré ŝ 10 », qui renvoie au Livre XII, X, Définitions, § 4, de l'édition de $1683^{9}$ :

4. Celles dont les angles, ou les côtés seraient alternativement égaux, c'est-à-dire le premier égal au $3^{\mathrm{e}}, 5^{\mathrm{e}}, 7^{\mathrm{e}}, 9^{\mathrm{e}}$, et le second au $4^{\mathrm{e}}, 6^{\mathrm{e}}, 8^{\mathrm{e}}, 10^{\mathrm{e}}$, se peuvent appeler alternativement équiangles ou équilatérales.

Mais il faut remarquer que cela ne peut être que quand le nombre des angles ou des côtés est pair. Car s'il était impair, le dernier et le premier se trouveraient égaux ; et par conséquent le pénultième et le premier seraient inégaux : et par conséquent ils ne seraient pas tous alternativement égaux. 
Il y a vraisemblablement eu une négociation, au terme de laquelle Arnauld, considérant que ce renvoi était un moindre mal, a dû accepter de supprimer une partie du texte, et de la remplacer par ce renvoi.

27 Mais l'affaire a retenti sur l'édition de 1683 : seize ans plus tard, Arnauld a obtenu le rétablissement du texte initial complet, assorti des deux figures pertinentes. Signe qu'il n’a jamais été satisfait de la rédaction abrégée. C'est aussi ce qui a eu lieu dans la grande édition de Genève.

\section{Une page d'errata retrouvée}

Cette double page n'est pas la seule pièce intéressante des Nouveaux éléments de géométrie de Clermont. On y trouve aussi une page libre additionnelle, que je n'ai rencontrée dans aucun des exemplaires que j'ai consultés, à l'exception de celui que présente le site e-rara de Zurich ${ }^{10}$.

Fig. 6. Page encartée

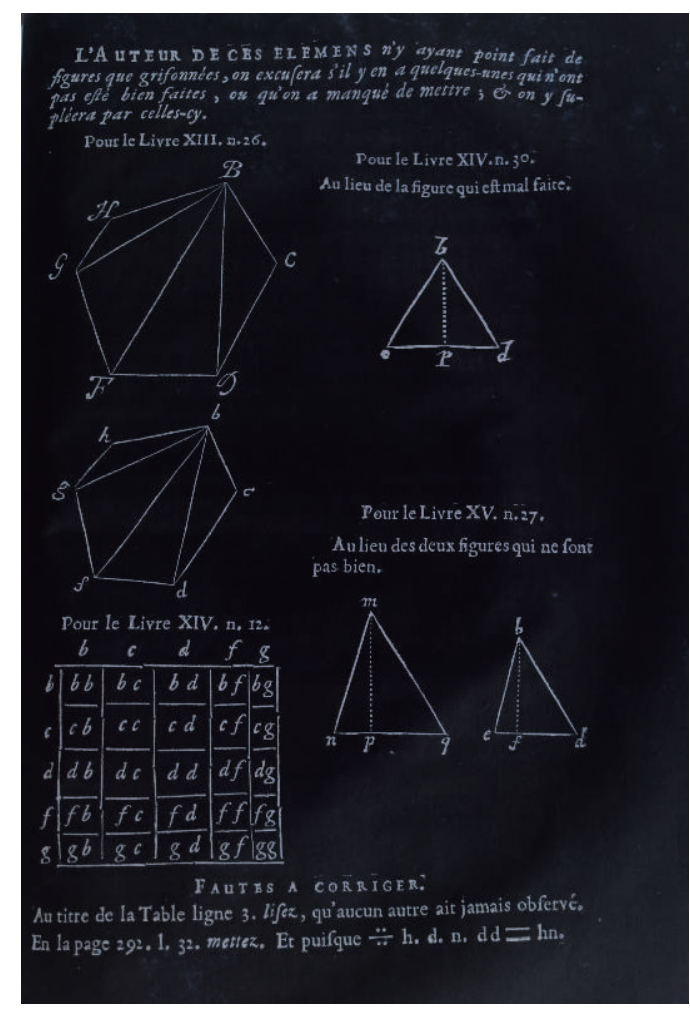

Bibliothèque du Patrimoine de Clermont-Métropole.

Ce feuillet a nécessairement dû être composé après les errata compris dans le volume. En revanche, il est difficile de dire s'il est antérieur ou postérieur à la substitution des pages décrite plus haut. En tout cas, il répondait à des réclamations tardives d'Arnauld qui n'ont pas été trop bien reçues par Savreux. Ces corrections portent sur les derniers livres, XIII, XIV et XV, ce qui paraît confirmer leur caractère tardif. Le fait que le feuillet a été encarté dans le volume explique sans doute qu'il n'ait pas été perdu, comme c'est le cas ailleurs. 
En tout cas, les réclamations d'Arnauld ont certainement indisposé Savreux, car les corrections sont précédées d'un texte qui manifeste une certaine irritation :

L'auteur de ces éléments n'y ayant point fait de figures que griffonnées, on excusera s'il y en a quelques-unes qui n'ont pas été bien faites, ou qu'on a manqué de mettre ; et on y suppléera par celles-ci.

Griffonnées, le terme est sévère. Mais il est vrai que ces fautes compliquent la lecture.

Les deux figures " pour le livre XIII, n. 26 ", renvoient à la p. 271 (p. 681 dans notre édition de 2009), où elles manquent effectivement, ce qui rend le texte difficile à suivre.

La figure « pour le livre XIV, n. 12 » renvoie à la p. 289 (p. 709 dans notre édition), où elle manque. Le texte est presque inintelligible.

34 La figure "pour le livre XIV, n. 30. Au lieu de la figure qui est mal faite », p. 294 (p. 717-718 de notre édition), remplace un triangle apparemment rectangle par un triangle équilatéral, conformément au texte.

5 Les figures «pour le livre XV, n. 27 ", « qui ne sont pas bien », renvoient à la p. 317 (p. 751 de notre édition), qui comportent des irrégularités vénielles.

Deux corrections textuelles sont ajoutées en bas de page.

Toutes ces rectifications ont été portées dans l'édition de 1683 . Une seule exception, assez surprenante : le tableau du Livre XIV, n. 12, manque dans l'impression de 1667. La lacune est corrigée en 1683. Mais alors que la figure des errata répond exactement aux corrections demandées par Arnauld, la deuxième édition n'en tient aucun compte, et reproduit la figure erronée de 1667, comme on peut en juger ci-dessous.

Fig. 7. État de l'édition de 1683

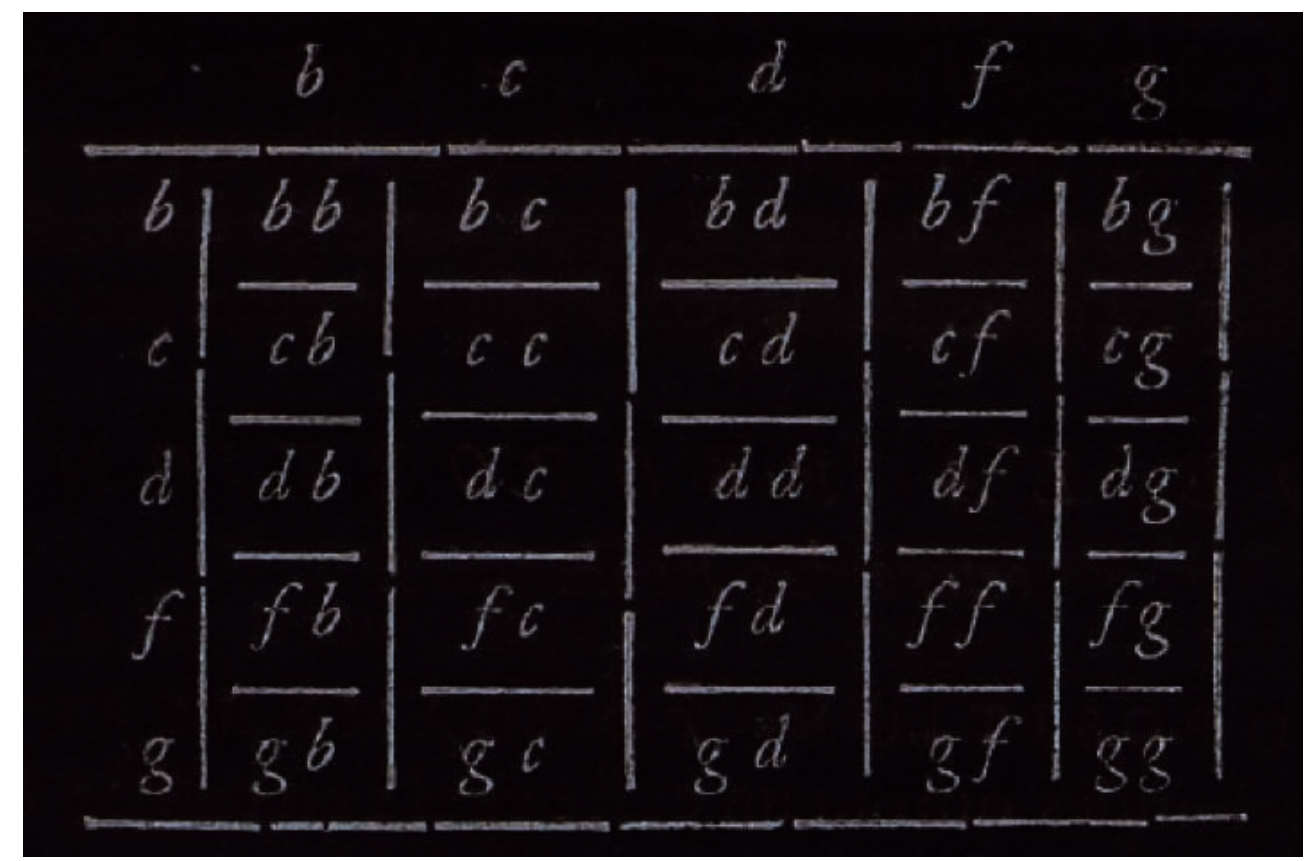

Bibliothèque du Patrimoine de Clermont-Métropole.

Cette différence semble avoir mécontenté Arnauld, qui s'en explique dans une lettre à une certaine $\mathrm{M}^{\mathrm{me}}$ Bois ${ }^{11}$.

Je n'ai reçu que depuis deux jours les nouveaux Éléments. Je les ai trouvés bien imprimés pour ce qui est du caractère. Mais n'en ayant lu que ce qui est de nouveau 
dans cette édition, j'y ai trouvé bien des fautes, outre celles de l'errata, que je voudrais qu'on eût mis en plus grosses lettres. Je ne m'en prends à personne. Je ne doute point que vous n'y ayez fait de votre mieux ; et je suis assuré que M. Ferrier y aura aussi mis un grand soin. Je lui en suis très obligé. Cela peut être venu du copiste qui a copié mes brouillons, qui n'a pas assez pris garde aux avis que j'avais donnés, et à suivre la ponctuation et les à linea. Car un des plus grands défauts est qu'il y en a trop peu. Quoi qu'il en soit, j'ai pensé au remède qu'on pourrait apporter à cela ; et je n'en ai point trouvé de meilleur que d'imprimer l'Avis au lecteur, que je vous envoie pour le mettre au commencement, afin que par là et l'errata, chacun puisse corriger son livre, comme j'ai été obligé d'en corriger un, avant que de le donner à une personne de condition de mes amis. Je salue ma commère et tout le reste de votre chère famille. Je prie Dieu qu'il la bénisse.

En ouvrant le livre, je suis tombé sur la page 347. Il y a une figure qu'on a voulu faire par des caractères d'impression, au lieu de la faire par une figure de bois; et elle est tout à fait mal faite ${ }^{12}$. Car au lieu qu'elle devait être carrée, c'est-à-dire aussi large d'un côté que de l'autre, elle est bien moins haute que large ; et les divisions sont presque égales, au lieu qu'elles devraient être notablement inégales.

Je viens encore de trouver une autre chose assez mal. C'est que dans le XIV livre on renvoie assez souvent au $2^{\mathrm{e}}$ et au $3^{\mathrm{e}}$ livre. Or comme ces deux livres sont tout changés, il fallait aussi changer ces renvois, et on a oublié de le faire. Je vous prie donc, mon compère, de faire imprimer l'avis que je vous envoie, qui réparera un peu des défauts de cet ouvrage, en donnant moyen de le corriger à ceux qui le voudront lire.

Je me suis encore aperçu qu'ayant ajouté au $\mathrm{X}^{\mathrm{e}}$ livre deux ou trois propositions touchant une ligne coupée harmoniquement, on ne les y a point mises, sans que j'en puisse deviner la raison; si ce n'est peut-être qu'on a eu si peu de soin des papiers que j'avais envoyés pour faire cette seconde édition, qu'on en ait laissé perdre cet endroit. Je souhaiterais qu'on le cherchât, et si on le trouvait, qu'on le fît imprimer avec ce titre, Addition pour la fin du $X^{e}$ livre, qui a été oubliée par mégarde.

Page 98, l. 24, propositions : lisez proportions.

Il faut encore ajouter que, dans l'exemplaire de Clermont, l'appendice sur les carrés magiques qui clôt les Nouveaux éléments comporte des erreurs bizarres. Les carrés naturels sont exacts. En revanche, les deux carrés magiques de XI et de XII comportent respectivement deux et quatre erreurs. Elles ont été corrigées à la main sur l'exemplaire même. Ces fautes sont d'autant plus surprenantes qu'elles ne se trouvent pas dans la plupart des exemplaires de l'édition de 1667.

Il n'y a guère que sur l'exemplaire de Zurich que j'aie pu constater des erreurs corrigées à la plume. Mais paradoxalement, il ne comporte que trois corrections sur le carré magique de XII, au lieu de 4. Peut-être est-ce la marque de corrections successives lors de la mise au point définitive. Ce qui impliquerait que l'exemplaire de Clermont est plus ancien que celui de Zurich. 
Fig. 8. Carré magique de XI

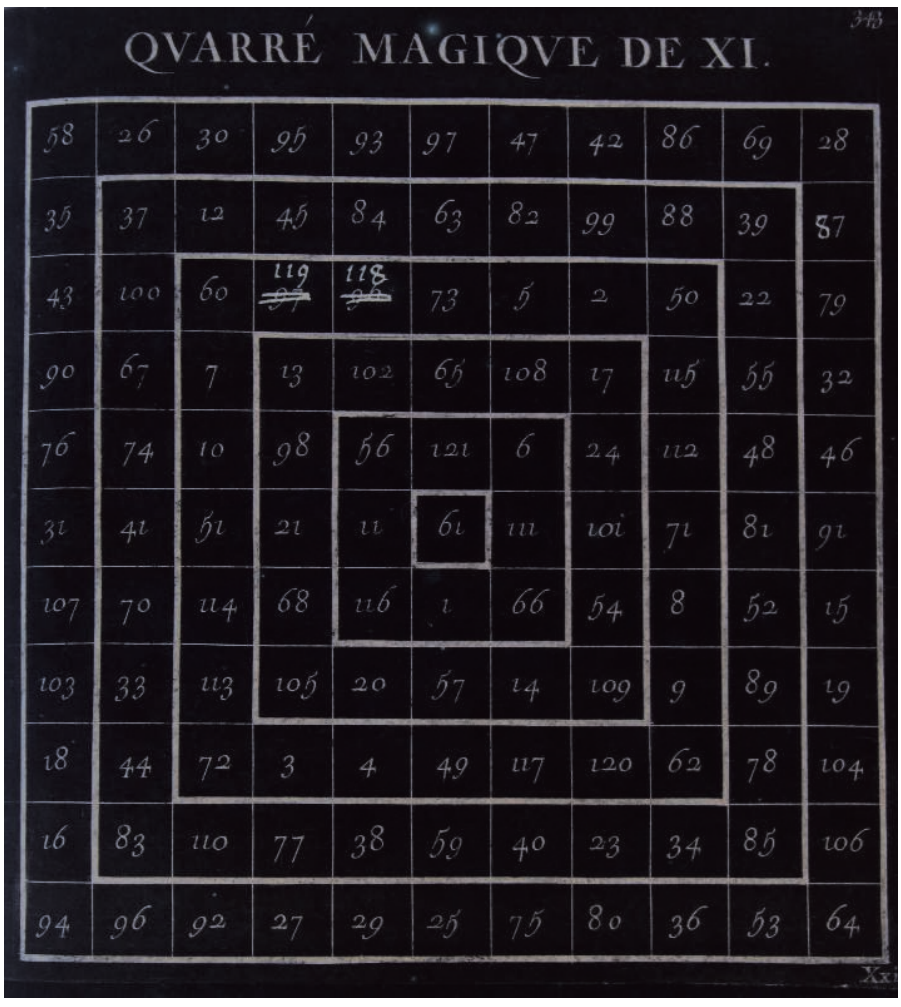

Bibliothèque du Patrimoine de Clermont-Métropole.

Fig. 9. Carré magique de XII

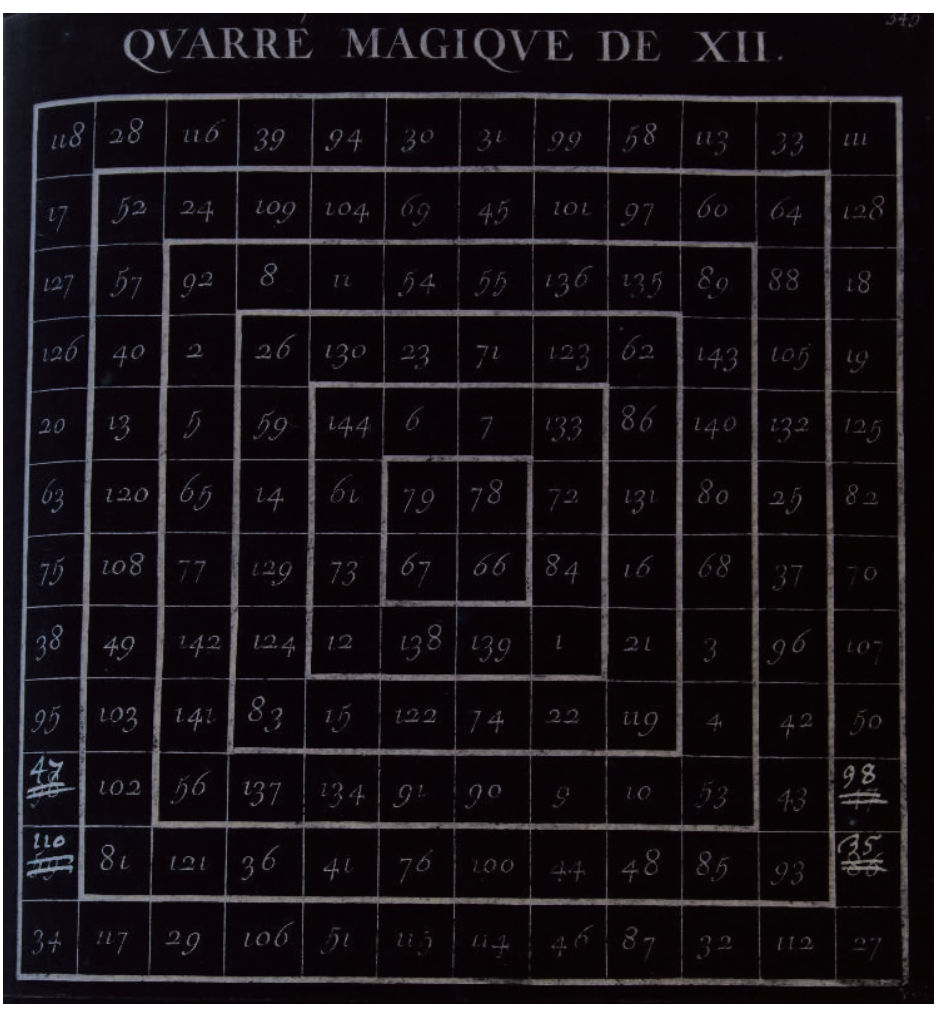

Bibliothèque du Patrimoine de Clermont-Métropole. 


\section{Conclusion}

L'exemplaire de Clermont fournit donc un état des Nouveaux éléments largement antérieur à la publication définitive. Il ne nous fournit évidemment pas tous les renseignements que l'on pourrait désirer sur la genèse de l'ouvrage. Mais il apporte d'intéressantes précisions sur les difficultés que pouvait rencontrer un auteur dans l'élaboration d'un manuel porteur d'innovations.

Comme à l'époque classique, la règle veut qu'une fois l'impression achevée, le manuscrit soit détruit, c'est par un bonheur rare que les péripéties de l'impression permettent de saisir quelques phases du processus génétique. On sait que Kokiti Hara, par exemple, est parvenu à trouver dans les écrits imprimés de Wallis la trace des fautes enfermées dans sa contribution initiale au concours de la roulette ${ }^{13}$. Les rares cas où l'on dispose aussi des manuscrits sont encore d'autant plus précieux qu'ils permettent d'observer sur le vif la transition de la plume à la presse ${ }^{14}$. Mais si ténues que soient les indications, elles sont toujours précieuses. L'histoire des sciences peut alors fructueusement s'appuyer sur les techniques de l'analyse littéraire ${ }^{15}$.

L'intérêt particulier des corrections apportées aux Nouveaux éléments de 1667, c'est qu'elles ne sont certainement l'effet du hasard ou de la malchance. En mathématiques, Arnauld n'est ni un génie du premier rang, ni un spécialiste. Pascal le dit à sa manière dans la Lettre à M. ADDS: la géométrie est pour lui un divertissement plutôt qu'une "étude ». Vraisemblablement, les figures soi-disant "griffonnées" doivent leurs imperfections au fait que qu'Arnauld a rarement eu affaire aux exigences graphiques de la géométrie. Arnauld a été l'un des esprits les plus puissants de son temps, mais il n'en avait pas moins un talon d'Achille en matière géométrique, qui transparaît çà et là dans ses Éléments. C'est d'autant plus regrettable que les Lettres de A. Dettonville de Pascal ou les Cylindrica et annularia du P. Tacquet montrent de quoi les graveurs étaient capables lorsqu'un vrai géomètre dirigeait leur travail.

Mais c'est précisément le fait que les mathématiques n'étaient pas son domaine propre qui lui a valu la dédicace de la Lettre à M. ADDS : son exemple montre qu'on peut être théologien et faire des mathématiques en honnête homme.

"L'homme est plein de besoins", dit Pascal. "Il n'aime que ceux qui peuvent les remplir tous. C'est un bon mathématicien dira-t-on, mais je n'ai que faire de mathématique; il me prendrait pour une proposition. C'est un bon guerrier: il me prendrait pour une place assiégée. Il faut donc un honnête homme qui puisse s'accommoder à tous mes besoins généralement ${ }^{16}$ ".

\section{NOTES}

1. Voir dans l'édition des Cuvres complètes de Pascal par J. Mesnard, III, Paris, Desclée de Brouwer, 1991, p. 429-434, la notice au Fragment d'introduction à la géométrie, et l'étude 
de J. Itard, «L'introduction à la Géométrie de Pascal », dans L'œuvre scientifique de Pascal, Centre International de Synthèse, Paris, Presses Universitaires de France, 1964, p. 102-119. Voir aussi les renseignements proposés dans mon édition des Nouveaux éléments de géométrie à la librairie Champion en 2009, sous le titre Géométries de PortRoyal, Paris, Champion, 2009, p. 22-23.

2. Le texte de cet état primitif de la Logique est reproduit dans l'édition de la Logique ou l'art de penser, éd. D. Descotes, Paris, Champion, 2011. Le Projet de nouveaux Éléments de géométrie se trouve dans la Partie IV, ch. IX, Réponses aux raisons que les géomètres apportent pour excuser ces défauts; il est publié dans mon édition de la Logique, Paris, Champion, 2011, p. 797-798.

3. L'édition des Nouveaux éléments de géométrie à la librairie Champion en 2009, sous le titre Géométries de Port-Royal, éd. D. Descotes, Paris, Champion, 2009.

4. Sur les modifications de fond apportées dans cette seconde édition, voir Jean-Louis Gardies, L'héritage épistémologique d'Eudoxe de Cnide. Un essais de reconstitution, Paris, Vrin, 1988, p. 125 sq.

5. Il existe des éditions tardives chez Van Bulderen (1690) et Van Duren (1711), qui n'ont pas lieu de nous retenir. Quant à l'édition des Euvres d'Arnauld imprimée à Lausanne par D'Arnay, elle écrase l'édition de 1667 sous celle de 1683, et surtout rejette toutes les figures dans des planches en fin de volume, ce qui rend impossible toute reconstitution de l'histoire des Éléments entre 1667 et 1683.

6. La Bibliothèque du Patrimoine possédait déjà l'édition de 1683 , lorsque la politique d'acquisition documentaire active conduite par son directeur, M. Guggenbuhl, a conduit à l'achat de l'édition de 1667. L'exemplaire a été présenté au public clermontois lors d'une exposition.

7. Ces précisions techniques ont été fournies par Julien Comellas, qui a mis le livre en vente.

8. Ce genre d'incident n'est pas rare : Pascal avait obtenu de Desprez qu'il remplace la rédaction initiale du Corollaire I de la Lettre à Carcavy par un texte de taille sensiblement double. Mais cela n'avait été possible qu'en resserrant la typographie sur plusieurs pages et en supprimant les passages à la ligne. Voir Pascal, Euvres complètes, IV, éd. J. Mesnard, Paris, Desclée de Brouwer, 1992, p. 428-431. Sur l'imprimé original, ce sont les pages 13-15. Voir sur ce point Descotes Dominique, « Genèse des Corollaires 1 et 2 de la Lettre à Carcavy de Blaise Pascal», Revue d'Histoire des Sciences, $\mathrm{n}^{\circ}$ 51/1, 1998, p. 127-138.

9. Voir la p. 303 (ou p. 647 de notre propre édition).

10. Voir l'adresse suivante : https://www.e-rara.ch/doi/10.3931/e-rara-13532.

11. Lettre à Mme Bois, dans Antoine Arnauld, Euvres, t. IV, p. 149-150, Appendice III. Lettre d'Antoine Arnauld à Madame Bois du 28 octobre 1683. Voir Géométries de PortRoyal, éd. D. Descotes, p. 786-787.

12. Voir livre XIV, Quatrième théorème, XII-XII [XII].

13. Kokiti Hara, L'CEuvre mathématique de Pascal, Osaka, 1981, p. 149-230.

14. Voir Dominique Descotes, "An Unknown Mathematical Manuscript by Blaise Pascal », Historia mathematica, vol. 37, 3, août 2010, p.503-534, sur l'unique cas de manuscrit mathématique contenu dans le manuscrit des Pensées. 
15. Voir notamment Kokiti Hara, L'Euvre mathématique de Pascal, Osaka, 1981; Jean Mesnard, CEuvres complètes de Pascal, II-IV, Paris, Desclée de Brouwer, 1970-1992 ; Claude Merker, Le calcul intégral dans la dernière œuvre scientifique de Pascal, I.R.E.M. de Besançon, Université de Franche-Comté, 1995 ; Jean-Louis Gardies, Pascal entre Eudoxe et Cantor, Vrin, Paris, 1984 ; Jean Itard, «L'Introduction à la géométrie de Pascal », dans L'œuvre scientifique de Pascal, Presses Universitaires de France, Paris, 1964, p. 102-119; les études de René Taton, «L'œuvre de Pascal en géométrie projective ", dans L'œuvre scientifique de Pascal, p. 17-72, et de Pierre Costabel, "Essai sur les secrets des Traités de la roulette », ibid., p. 169-206 ; Dominique Descotes, Blaise Pascal. Littérature et géométrie, ClermontFerrand, Presses Universitaires Blaise Pascal, 2001. Une belle thèse de Joao Cortese sur l'idée de disproportion chez Pascal sera publiée prochainement.

16. Pensées, Laf. 605, Sel. 502.

INDEX

Mots-clés : géométrie, mathématiques, Arnauld (Antoine)

Keywords : geometry, mathematics, Arnauld (Antoine)

\section{AUTEUR}

DOMINIQUE DESCOTES

IHRIM, Université Clermont Auvergne 